\title{
Cerebellum-Editorial Regarding Consensus Paper Consensus on Virtual Management of Vestibular Disorders: Urgent Versus Expedited Care. Shaikh et al., doi.org/10.1007/s12311-020—01178-8
}

\author{
The Return of the House Call: Evaluating Acutely III Patients with Vertigo in the Era of Virtual \\ Health Care
}

\author{
Janet C. Rucker ${ }^{1}$ - David S. Zee ${ }^{2}$ \\ Published online: 2 September 2020 \\ (C) Springer Science+Business Media, LLC, part of Springer Nature 2020
}

The SARS-CoV-2 pandemic has affected almost every aspect of how we care for patients, including the evaluation of patients with new neurological symptoms. Telemedicine and the remote evaluation have become increasingly part of conventional medical practice, but with the pandemic, telemedicine has suddenly become the way neurologists identify most of the patients with acute neurological disorders who must go immediately to the hospital for an in-person evaluation. For all physicians, the patient with the new onset of dizziness is particularly difficult, given that dizziness is a common symptom [1] with a broad differential diagnosis that includes both benign and serious causes. Especially vexing are patients with the acute vestibular syndrome (AVS) with rapid-onset vertigo and/or unsteadiness often accompanied by nausea, vomiting, and motion intolerance. Differentiation between common benign causes, such as vestibular neuritis and benign paroxysmal positional vertigo, and less common but potentially lifethreatening neurological emergencies, such as brainstem or cerebellar stroke, requires a detailed and often timeconsuming ocular motor and vestibular examination. Furthermore, the pathological signs are often subtle and require familiarity with the underlying physiology and the skill to elicit and properly interpret them.

Janet C. Rucker

janet.rucker@nyulangone.org

1 Departments of Neurology and Ophthalmology, NYU Grossman School of Medicine, 222 East 41st Street, 14th Floor, New York, NY 10017, USA

2 Departments of Neurology, Otolaryngology-Head and Neck Surgery, Ophthalmology and Neuroscience, Johns Hopkins University School of Medicine, Baltimore, MD, USA
The need for more accurate diagnostic tools and decisionmaking algorithms for patients with the AVS presenting to the emergency department has received much recent attention [2, 3]. Even with a face-to-face examination of patients with AVS, there is under-recognition of peripheral vestibular syndromes not requiring neuroimaging [4-6], uncertainty with regard to when neuroimaging is necessary [3], and a high diagnostic error rate including missed strokes [7-9]. When performed by physicians with specialized vestibular training, a three-step bedside ocular motor examination incorporating the high-velocity vestibular head impulse test, evaluation for unidirectional versus direction-changing nystagmus, and testing for a vertical misalignment of the eyes (skew deviation) due to asymmetric processing of otolith information (e.g., HINTS: Head Impulse, Nystagmus, Test of Skew) has been shown to be superior to early MRI diffusion-weighted imaging [10] and to a standard stroke risk stratification rule (ABCD2: age, blood pressure, clinical features, duration of symptoms, diabetes) [11] for detection of stroke in AVS. HINTS suggests a central lesion when the head impulse test appears normal without corrective saccades, nystagmus is direction-changing (the fast phases of nystagmus change direction with changes in gaze direction, for example, right beat nystagmus in right gaze and left beat nystagmus in left gaze), and when a skew deviation is present. Adding a change in hearing on one side and an inability to walk improves the accuracy of the HINTS algorithm in deciding which patients might be having a stroke. Proper application and interpretation of HINTS, however, is challenging for the non-specialist since it requires experience and the findings can be subtle. Even for the trained neurotologist, the sensitivity and specificity of bedside HINTS is enhanced with oculographic recordings of the head impulse test and the patterns of nystagmus, since abnormalities below clinical perception at the bedside can only be detected with recordings [12]. 
Prior to SARS-CoV-2, telemedicine in neurology was largely restricted to remote evaluation of a patient by a subspecialist in very specific clinical scenarios, including for the immediate management of stroke $[13,14]$. The diagnostic accuracy of remote interpretation of eye movement examinations and recordings obtained in the emergency department in patients with AVS by vestibular subspecialists are being evaluated. For example, the currently recruiting AVERT (Acute Video-oculography for Vertigo in Emergency Rooms for Rapid Triage) trial is a multicenter, phase II clinical trial comparing standard emergency department care for AVS patients with an algorithm-determined diagnosis based on an automated video-oculographic recording interpretation [15]. Such studies have been made feasible by technological advances in video-oculographic cameras that have made portable eye movement recording accessible and by specific development and validation of goggle VOG-based head impulse test devices $[16,17]$. In addition to clinical trials, "Tele-Dizzy" services were created to allow for video-oculographic HINTS examinations and positional testing for benign paroxysmal positional vertigo obtained in the emergency room by a trained technician and then interpreted remotely by a vestibular subspecialist.

In the global crisis of the SARS-CoV-2 pandemic, the practices of many neurologists across the world changed instantaneously to the model of providing most care by virtual assessment of patients via telemedicine. Neurologists struggled to determine how best to perform their routine but complex, detailed, and time-consuming examinations virtually. They were forced to identify patients in need of emergency room evaluation or urgent neuroimaging in an environment in which patients feared hospitals and were hesitant to leave their homes. "Consensus on Virtual Management of Vestibular Disorders: Urgent Versus Expedited Care" [18], by Shaikh and a group of vestibular experts from around the globe, provides a survival guide of best practices geared towards the non-vestibular specialist evaluating patients with AVS remotely on virtual platforms. The objectives are two-fold: to provide tips on how to conduct an eye movement/vestibular examination on telemedicine platforms and to help clinicians to decide when an immediate evaluation in the emergency department is needed to rule out stroke based on the history and findings on examination findings obtained in the virtual visit. A baseline knowledge of aspects of the specialized ocular motor and vestibular examination is required and can be found elsewhere [19]. One must emphasize, as the consensus paper authors do, that when there is doubt caused by clinical uncertainty in interpretation of the examination or poor quality of the virtual visit, the patient with an AVS should be directed to the emergency department for immediate additional evaluation. The inability of the patient to cooperate for the examination because of fatigue, nausea and vomiting, discomfort, as well as technical and practical issues from poor lighting, slow
Wi-Fi connections, and limited experience with technology, all may limit what can be learned from the virtual visit. We emphasize, as did Shaikh et al., that we do not yet know which history taking and examination techniques will be shown to be useful and practical in the telemedicine visit. Examination of acutely ill patients with vertigo is often difficult even in the well-equipped office or hospital setting, and what might be accomplished remotely needs to be carefully validated.

Even with these caveats, we share the enthusiasm for the increasing role of telemedicine in managing the acutely dizzy patient and recognize that even when the pandemic is over, there will be no going back to the old model of always seeing the patient in the hospital or office. We emphasize that telemedicine is bringing us back to a more old-fashioned type of bedside clinical medicine. The skill of history taking has become even more important, since time constraints, the inability of sick patients to cooperate, and the limitations of being remote require that a differential diagnosis of probable causes be rapidly developed based on a targeted history, which in turn guides a relatively brief, focused examination [20]. For example, Kim et al. recently showed how the answers to just a few questions markedly improves the ability to diagnose benign paroxysmal positional vertigo, to decide which canal is affected, and to know with which maneuver to treat the particular affected canal [21]. Similarly, the properly performed HINTS examination takes less than a few minutes and positional testing when necessary just a few minutes more. The choice of whether to image or not and which image to obtain is best guided by the results of the focused history and the HINTS evaluation. Medical students and physicians in many disciplines must be taught the modern day skills of history taking and examination of the dizzy patient. Somewhat paradoxically, telemedicine and new technologies are forcing us to become better old school physicians as we again rely on a careful history and we resume making old-fashioned "house calls."

\section{References}

1. Kerber KA, Meurer WJ, West BT, Fendrick AM. Dizziness presentations in U.S. emergency departments, 1995-2004. Acad Emerg Med. 2008;15:744-50.

2. Newman-Toker DE, Kattah JC, Alvernia JE, Wang DZ. Normal head impulse test differentiates acute cerebellar strokes from vestibular neuritis. Neurology. 2008;70:2378-85.

3. Eagles D, Stiell IG, Clement CM, Brehaut J, Kelly AM, Mason S, et al. International survey of emergency physicians' priorities for clinical decision rules. Acad Emerg Med. 2008;15:177-82.

4. Newman-Toker DE, Camargo CA Jr, Hsieh YH, Pelletier AJ, Edlow JA. Disconnect between charted vestibular diagnoses and emergency department management decisions: a cross-sectional analysis from a nationally representative sample. Acad Emerg Med. 2009;16:970-7.

5. Kerber KA, Morgenstern LB, Meurer WJ, McLaughlin T, Hall PA, Forman J, et al. Nystagmus assessments documented by emergency 
physicians in acute dizziness presentations: a target for decision support? Acad Emerg Med. 2011;18:619-26.

6. Kerber KA, Burke JF, Skolarus LE, Meurer WJ, Callaghan BC, Brown DL, et al. Use of BPPV processes in emergency department dizziness presentations: a population-based study. Otolaryngol Head Neck Surg. 2013;148:425-30.

7. Savitz SI, Caplan LR, Edlow JA. Pitfalls in the diagnosis of cerebellar infarction. Acad Emerg Med. 2007;14:63-8.

8. Kuruvilla A, Bhattacharya P, Rajamani K, Chaturvedi S. Factors associated with misdiagnosis of acute stroke in young adults. J Stroke Cerebrovasc Dis. 2011;20:523-7.

9. Newman-Toker DE, Moy E, Valente E, Coffey R, Hines AL. Missed diagnosis of stroke in the emergency department: a crosssectional analysis of a large population-based sample. Diagnosis (Berl). 2014;1:155-66.

10. Kattah JC, Talkad AV, Wang DZ, Hsieh YH, Newman-Toker DE. HINTS to diagnose stroke in the acute vestibular syndrome: threestep bedside oculomotor examination more sensitive than early MRI diffusion-weighted imaging. Stroke. 2009;40:3504-10.

11. Newman-Toker DE, Kerber KA, Hsieh YH, Pula JH, Omron R, Saber Tehrani AS, et al. HINTS outperforms ABCD2 to screen for stroke in acute continuous vertigo and dizziness. Acad Emerg Med. 2013;20:986-96.

12. Weber KP, Aw ST, Todd MJ, McGarvie LA, Curthoys IS, Halmagyi GM. Head impulse test in unilateral vestibular loss: vestibulo-ocular reflex and catch-up saccades. Neurology. 2008;70:454-63.

13. Amarenco P. "Telethrombolysis": stroke consultation by telemedicine. Lancet Neurol. 2008;7:763-5.
14. Schwamm LH, Audebert HJ, Amarenco P, Chumbler NR, Frankel MR, George MG, et al. Recommendations for the implementation of telemedicine within stroke systems of care: a policy statement from the American Heart Association. Stroke. 2009;40:2635-60.

15. Newman-Toker DE. (2015) AVERT Phase II Trial: Acute videooculography for vertigo in emergency rooms for rapid triage. https://clinicaltrials.gov/ct2/show/NCT02483429.

16. Weber KP, MacDougall HG, Halmagyi GM, Curthoys IS Impulsive testing of semicircular-canal function using videooculography. Ann N Y Acad Sci. 2009;1164:486-91.

17. Bartl K, Lehnen N, Kohlbecher S, Schneider E. Head impulse testing using video-oculography. Ann N Y Acad Sci. 2009;1164:3313.

18. Shaikh AG, Bronstein A, Carmona S, Cha YH, Cho C, Ghasia FF, et al. Consensus on virtual management of vestibular disorders: urgent versus expedited care. Cerebellum. 2020. https://doi.org/ 10.1007/s12311-020-01178-8.

19. Shemesh AA, Gold DR. Dizziness and vertigo: the skillfull examination. J Neuroophthalmol. 2020;40:e49-61.

20. Edlow JA, Gurley KL, Newman-Toker DE. A new diagnostic approach to the adult patient with acute dizziness. J Emerg Med. 2018;54:469-83.

21. Kim HJ, Song JM, Zhong L, Yang X, Kim JS. Questionnaire-based diagnosis of benign paroxysmal positional vertigo. Neurology. 2020;94:e942-9.

Publisher's Note Springer Nature remains neutral with regard to jurisdictional claims in published maps and institutional affiliations. 